Pure and Applied Mathematics Quarterly

Volume 2, Number 1

(Special Issue: In honor of

John H. Coates, Part 1 of 2$)$

$147-161,2006$

\title{
On Hessian Measures for Non-Commuting Vector Fields
}

\author{
Neil S Trudinger
}

\begin{abstract}
Previous results on Hessian measures by Trudinger and Wang are extended to the subelliptic case. Specifically we prove the weak continuity of the 2-Hessian operator, with respect to local $L^{1}$ convergence, for a system of $m$ vector fields of step 2 and derive gradient estimates for the corresponding $k$-convex functions, $1 \leq k \leq m$.
\end{abstract}

\section{INTRODUCTION}

In the paper [18], we introduced the notion of $k$-convexity, $k=1 \ldots n$, for functions $u$ defined on domains $\Omega$ in Euclidean space, $\mathbb{R}^{n}$. Namely, for $u \in C^{2}(\Omega)$, we call $u k$-convex in $\Omega$ if

$$
F_{j}[u]:=F_{j}\left(D^{2} u\right):=S_{j}(\lambda) \geq 0,
$$

for $j=1 \ldots k$, where $\lambda=\left(\lambda_{1}, \ldots, \lambda_{n}\right)$ are the eigenvalues of the Hessian matrix $D^{2} u$ of second derivatives of $u$ and $S_{j}$ denotes the $j$ th elementary symmetric function, that is

$$
S_{j}(\lambda)=\sum_{i_{1}<\ldots<i_{j}} \lambda_{i_{1}} \ldots \lambda_{i_{j}}, \quad j=1 \ldots n .
$$

When there is no confusion we use the same notation $F_{j}$ for both the operator and the function on $\mathbb{R}^{n} \times \mathbb{R}^{n}$. Equivalently, $u$ is $k$-convex in $\Omega$ if $u$ is subharmonic with respect to the operator $F_{k}$ and this is the basis for our definition of $k$-convexity for non-smooth functions in our sequel papers [19], [20], [21]. The core result in our paper [19], is that the mapping $u \mapsto F_{k}[u]$ is weakly continuous as a mapping from $L_{l o c}^{1}(\Omega)$ to $M_{l o c}(\Omega)$, the space of locally finite measures in $\Omega$, that is for any

Received May 26, 2005.

Research supported by Australian Research Council grant. 
subdomain $\Omega^{\prime} \subset \subset \Omega, \eta \in C_{0}^{0}\left(\Omega^{\prime}\right)$ and positive constant $\epsilon$, there exists a constant $\delta$ such that

$$
\left|\int_{\Omega} \eta\left(F_{k}(u)-F_{k}(v)\right)\right|<\epsilon
$$

whenever

$$
\int_{\Omega^{\prime}}|u-v|<\delta, \quad \int_{\Omega^{\prime}}|u+v|<1
$$

for arbitrary $k$-convex $u$ and $v$. This result enables us to define for any locally integrable $k$-convex function $u$, the Hessian measure, $\mu_{k}[u]$, as an extension of $F_{k}[u]$. In our first paper [18], we only proved the continuity of $\mu_{k}$ from $C^{0}(\Omega)$ to $M_{l o c}(\Omega)$ but this was enough for the cases $k>\frac{n}{2}$, which included the MongeAmpére measure when $k=n$. The weak continuity of the Monge-Ampére measure is a fundamental result of Aleksandrov (see eg. [16]).

In this paper we extend our results in [19] to the case of non-commuting vector fields but only prove the corresponding weak continuity for the case $k=2$. Our approach follows [19], with some help from [20] and [21], and is inspired by the recent paper [8] on the special case of the Heisenberg group $\mathbb{H}^{1}$, by Gutiérrez and Montanari, where the more restrictive approach in [18] was adequate. To formulate the main theorem, we let $X=X_{1} \ldots X_{m}$ denote a system of vector fields in $\mathbb{R}^{n}$, that is first order differential operators of the form

$$
X_{i}=\sum_{j=1}^{n} b^{i j} D_{j}
$$

with coefficients $b^{i j} \in C^{\infty}(\bar{\Omega})$ (although weaker regularity will suffice). Then, for $k=1 \ldots m$, we call a function $u \in C^{2}(\Omega), k$-convex, with respect to $X$ if

$$
F_{j}[u]:=F_{j}\left(X_{s}^{2} u\right):=S_{j}(\lambda) \geq 0, \quad j=1 \ldots k
$$

where now $\lambda=\left(\lambda_{1} \ldots \lambda_{m}\right)$ denote the eigenvalues of the symmetric Hessian,

$$
X_{s}^{2} u=\left[\frac{1}{2}\left(X_{i} X_{j}+X_{j} X_{i}\right) u\right]_{i, j=1 \ldots m} .
$$

Our hypotheses on the vector fields $X_{1} \ldots X_{m}$ are that:

(i) they are anti-self adjoint, namely

$$
X_{i}^{*}=-X_{i}, \quad i=1 \ldots m
$$

(ii) they satisfy the Hormander condition, namely the Lie algebra generated by them spans $\mathbb{R}^{n}$ and

(iii) the second commutators formed from any two vector fields vanish.

These conditions will be automatically satisfied by the vector fields generating an homogeneous group of Heisenberg type. We can now state the main theorem. 
Theorem 1.1. The mappings

$$
u \mapsto F_{2}[u]+\alpha \sum_{i<j}\left[X_{i}, X_{j}\right]^{2} u,
$$

for u 2-convex in $\Omega$, are weakly continuous from $L_{l o c}^{1}(\Omega)$ to $M_{l o c}(\Omega)$, for any constant $\alpha$.

As mentioned above, the special case of the Heisenberg group $\mathbb{H}^{1}$, given by

$$
\begin{aligned}
X_{1} u & =D_{1} u-\frac{1}{2} x_{2} D_{3} u, \\
X_{2} u & =D_{2} u+\frac{1}{2} x_{1} D_{3} u, \\
{\left[X_{1}, X_{2}\right] u } & =D_{3} u,
\end{aligned}
$$

is proved in [8]. Here $L_{l o c}^{1}(\Omega)$ convergence is equivalent to local uniform convergence in $\Omega$ and the proof is much simpler.

Theorem 1.1 enables us to assign a Borel measure $\mu_{2}[u]$ to any $L_{l o c}^{1}(\Omega)$ limit of smooth 2-convex functions, which extends $F_{2}[u]$ and is weakly continuous. Letting $\Phi^{2}(\Omega)$ denote the space of such functions we also see that the commutators $\left[X_{i}, X_{j}\right] u \in L_{l o c}^{2}(\Omega)$ for $u \in \Phi^{2}(\Omega)$.

This paper is arranged as follows. In the next section we generalize the basic divergence identity of Gutiérrez and Montanari [8], [9] on Heisenberg groups to vector fields satisfying conditions (i) and (iii). A more complete treatment in Carnot groups, with applications to monotonicity, is given by Danielli, Garofalo, Nhieu and Tournier in [5]; (see also [7]). In Section 3, we employ our approach in [19] to obtain integral estimates for the subelliptic gradient $X u$ under conditions (i) and (ii), Theorem 3.1. As we cannot extend all of our argument in [19], we have to rely strongly on the subelliptic potential estimates in [21]. In Section 4, we carry out the arguments, again adapting [19] to the non-commutative case, to conclude the local boundedness and weak continuity of the functionals in Theorem 1.1, thereby completing the proof. Finally in Section 5, we extend our previous results to the classes $\phi^{k}(\Omega)$ of $L_{l o c}^{1}$ limits of $k$-convex functions. Additional remarks at the ends of Sections 4 and 5 treat the removal of condition (iii) and more general definitions of $k$-convexity.

We are grateful for useful comments and discussions with N. Chaudhuri, X-J Wang, A. Montanari and T. Nguyen.

\section{Divergence structure And monotonicity}

It is well known that if the vector fields $X_{1} \ldots X_{m}$ commute, then

$$
X_{i} F_{k}^{i j}\left(X^{2} u\right)=0, \quad j=1 \ldots m,
$$


where

$$
X^{2} u=X_{s}^{2} u=\left[X_{i} X_{j} u\right]_{i, j=1 \ldots m}
$$

and

$$
F_{k}^{i j}(r):=\frac{\partial}{\partial r_{i j}} F_{k}(r)
$$

The identity (2.1), which means that the columns of the linearized coefficient matrix (2.3) are divergence free, was the basis for our approach in [18], [19]. Now suppose, more generally, that the second commutators formed from any two vector fields vanish, that is for any $i, j=1 \ldots m$,

$$
\begin{aligned}
0 & =\left[X_{i},\left[X_{i}, X_{j}\right]\right] \\
& =X_{i}\left[X_{i}, X_{j}\right]-\left[X_{i}, X_{j}\right] X_{i} \\
& =X_{i} X_{i} X_{j}-2 X_{i} X_{j} X_{i}+X_{j} X_{i} X_{i} \\
& =X_{j}\left(X_{i} X_{i}\right)+X_{i}\left(X_{i} X_{j}-2 X_{j} X_{i}\right) .
\end{aligned}
$$

Then, defining for any real matrix $r \in \mathbb{R}^{n} \times \mathbb{R}^{n}$,

$$
\begin{aligned}
\mathscr{F}_{2}(r) & :=\frac{1}{2}\left\{\left(r_{i i}\right)^{2}-r_{i j} r_{j i}+\frac{1}{2}\left(r_{i j}-r_{j i}\right)^{2}\right\} \\
& =F_{2}[r]+\frac{3}{4} \sum_{i<j}\left(r_{i j}-r_{j i}\right)^{2},
\end{aligned}
$$

we have the identity

$$
X_{i} \mathscr{F}_{2}^{i j}\left(X^{2} u\right)=0
$$

where

$$
\begin{aligned}
\mathscr{F}_{2}^{i j}(r) & =\frac{\partial \mathscr{F}_{2}}{\partial r_{i j}}(r) \\
& =(\text { trace } r) \delta_{i j}+r_{i j}-2 r_{j i},
\end{aligned}
$$

which extends (2.1) in the case $k=2$. The identity (2.5) was discovered by Gutiérrez and Montanari [8], [9] for the Heisenberg groups $\mathbb{H}^{n}$, (see also [5], [7]). From (2.5), we infer the monotonicity formula for the operator $\mathscr{F}_{2}$ defined by

$$
\mathscr{F}_{2}[u]=\mathscr{F}_{2}\left(X^{2} u\right),
$$

extending Lemma 2.1 in [18] for $k=2$.

Lemma 2.1. Let $u, v \in C^{2}(\Omega) \cap C^{0}(\bar{\Omega})$ satisfy $u \leq v$ in $\Omega, u=v$ on $\partial \Omega$ with the operator $F_{2}$ degenerate elliptic with respect to their sum $u+v$, that is

$$
\begin{aligned}
\mathscr{F}_{2}^{i j}\left(X^{2}(u+v)\right) \xi_{i} \xi_{j} & =F_{2}^{i j}\left(X^{2}(u+v)\right) \xi_{i} \xi_{j} \\
& \geq 0
\end{aligned}
$$


for all $\xi \in \mathbb{R}^{m}$. Then, if the vector fields $X_{1} \ldots X_{m}$ satisfy conditions (i) and (iii), we have

$$
\int_{\Omega} \mathscr{F}_{2}[v] \leq \int_{\Omega} \mathscr{F}_{2}[u]
$$

Proof. By integration by parts and the identity (2.5), we have, for $u, v \in C^{2}(\bar{\Omega})$,

$$
\begin{aligned}
\int_{\Omega}\left(\mathscr{F}_{2}[u]-\mathscr{F}_{2}[v]\right) & =\int_{0}^{1} d t \int_{\Omega} \mathscr{F}_{2}^{i j}\left[X^{2}(t u+(1-t) v)\right] X_{i} X_{j}(u-v) \\
& =\int_{0}^{1} d t \int_{\partial \Omega} \mathscr{F}_{2}^{i j}\left(X_{i} \cdot \gamma\right) X_{j}(u-v) \\
& =\int_{0}^{1} d t \int_{\partial \Omega} \mathscr{F}_{2}^{i j}\left(X_{i} \cdot \gamma\right)\left(X_{j} \cdot \gamma\right)|D(u-v)| \\
& \geq 0
\end{aligned}
$$

Here $\gamma$ denotes the outer unit normal to $\partial \Omega$ and

$$
X_{i} \cdot \gamma=b^{i j} \gamma_{j}
$$

The general case $u, v \in C^{0}(\bar{\Omega}) \cap C^{2}(\Omega)$ follows by approximation.

More general version of Lemma 2.1 are presented in [5]. For weak continuity with respect to $C^{0}(\Omega)$ and for groups of Heisenberg type we may proceed exactly as in [18]. In the next section we present the basic gradient estimates for $k$ convex functions needed to handle the general case, for which we will not need Lemma 2.1.

\section{Gradient Estimates}

In this section we provide the necessary gradient estimates for our proof of weak continuity. For these we do not have to restrict to the case $k=2$ and moreover we only need to assume the vector fields $X_{1} \ldots X_{m}$ satisfy conditions (i) and (ii). First we note that since $k$-convexity implies 1 -convexity, $k$-convex functions $u$ are subharmonic with respect to the sub-Laplacian associated with $X_{1} \ldots X_{n}$, that is

$$
\Delta_{X} u:=X_{i} X_{i} u \geq 0
$$

in $\Omega$. From (3.1) we infer immediately a bound from above, namely, for any $\Omega^{\prime} \subset \subset \Omega$,

$$
\sup _{\Omega^{\prime}} u \leq C \int_{\Omega}|u|
$$

where the constant $C$ depends on $X_{1} \ldots X_{m}$ and $\operatorname{dist}\left(\Omega^{\prime}, \partial \Omega\right)$. As we are only dealing with local estimates in this paper, we will always assume, without loss of 
generality, that $u \in L^{1}(\Omega)$. Following [19], our treatment of gradient estimates depends on the relation between $k$-convexity and the subelliptic p-Laplacian operators $\Delta_{p}$ defined by

$$
\Delta_{p} u=X_{i}\left(|X u|^{p-2} X_{i} u\right)
$$

for $p>1$.

Lemma 3.1. Let $u$ be $k$-convex in $\Omega$. Then $u$ is subharmonic with respect to $\Delta_{p}$ for $p-1 \leq k(m-1) /(m-k)$.

Proof. Although this is just the special case $l=1$ in Lemma 4.2 of [19], we include it for completeness as it is simpler than the cases $l>1$. We use the notation

$$
S_{k, i}(\lambda)=\left.S_{k}(\lambda)\right|_{\lambda_{i}=0}
$$

so that

$$
S_{j, i}(\lambda) \geq 0
$$

for all $j \leq k-1$, if $S_{j}(\lambda) \geq 0$, for all $j=1, \ldots k$, [19]. It follows then that

$$
0 \leq S_{k}(\lambda)=S_{k, i}(\lambda)+S_{k-1, i}(\lambda) \lambda_{i}, \quad i=1, \ldots m,
$$

whence

$$
\begin{aligned}
-\lambda_{i} & \leq \frac{S_{k, i}}{S_{k-1, i}}(\lambda) \\
& \leq \frac{(m-k)}{k(m-1)} S_{1, i}(\lambda)
\end{aligned}
$$

by MacLaurins' inequality for ratios of elementary symmetric functions. Consequently, if $u$ is $k$-convex,

$$
\begin{aligned}
\Delta_{p} u & =X_{i}\left(|X u|^{p-2} X_{i} u\right) \\
& =|X u|^{p-2}\left\{\Delta_{X} u+(p-2) \frac{X_{i} u X_{j} u}{|X u|^{2}} X_{i} X_{j} u\right\} \\
& \geq|X u|^{p-2}\left\{\Delta_{X} u+(p-2) \lambda_{\min }\left(X_{s}^{2} u\right)\right\} \\
& \geq 0,
\end{aligned}
$$

for $p-1 \leq k(m-1) /(m-k)$, by taking $\lambda_{1} \ldots \lambda_{m}$ in (3.7) to be the eigenvalues of $X_{s}^{2} u$.

Note that Lemma 3.1 also includes the case $k=m, p=\infty$, when $\Delta_{p}$ is the subelliptic $\infty$-Laplacian

$$
\Delta_{\infty} u=X_{i} u X_{j} u X_{i j} u
$$

Our gradient estimates now follow immediately from [21] but to express them we need the concept of homogeneous dimension. For our purposes here, we define 
the C-C (Carnot-Caratheodory) metric induced from the vector fields $X_{1} \ldots X_{m}$ by

$$
\begin{gathered}
d(x, y)=\inf \left\{T>0 \mid \exists \text { a sub-unitary } \gamma:[0, T] \rightarrow \mathbb{R}^{n}\right. \\
\text { with } \gamma(0)=x, \gamma(T)=y\}
\end{gathered}
$$

where a piecewise $C^{1}$ curve $\gamma:[0, T] \rightarrow \mathbb{R}^{n}$ is said to be sub-unitary, with respect to $X_{1} \ldots X_{m}$, if for every $\xi \in \mathbb{R}^{n}$ and $t \in(0, T)$,

$$
\left|\gamma^{\prime}(t) . \xi\right|^{2} \leq \sum_{i=1}^{m}\left(X_{i}(\gamma(t)) \cdot \xi\right)^{2} .
$$

Let $B_{R}(x)$ denote the C-C ball $\left\{y \in \mathbb{R}^{n} \mid d(x, y)<R\right\}$, and let $\Omega$ be a bounded domain in $\mathbb{R}^{n}$. The the fundamental result of Nagel, Stein and Wainger [15] asserts that there exist positive constants $C, R_{0}$ and positive integer $Q$, depending on $X$ and $\Omega$ such that

$$
\left|B_{t R}(x)\right| \geq C t^{Q}\left|B_{R}(x)\right|
$$

for any $x \in \Omega, t \in(0,1)$ and $R<R_{0}$, where $\|$ denotes the Lebesgue volume. The number $Q(\geq n)$, is chosen as the least integer for which (3.12) holds and is called the homogeneous dimension of $X$ in $\Omega$. For (3.12) we only need the Hormander condition (ii) and we could replace it more generally in this paper by simply the validity of (3.12).

Theorem 3.1. For any $k$-convex function $u$ in $\Omega$, and subdomain $\Omega^{\prime} \subset \subset \Omega$, we have the estimates

$$
\begin{gathered}
\|X u\|_{L^{q}\left(\Omega^{\prime}\right)} \leq C\left(\int_{\Omega}|u|\right) \\
\int_{\Omega^{\prime}}|X u|^{r} \Delta_{X} u \leq C\left(\int_{\Omega}|u|\right)^{1+r}
\end{gathered}
$$

for $1 \leq q<Q k(m-1) /(Q-1)(m-k), 0 \leq r<m(k-1) /(m-k)$ where $C$ depends on $\Omega, \Omega^{\prime}, X_{1} \ldots X_{m}$ and $q$ or $r$ as appropriate.

Proof. The estimate (3.13) follows from Lemmas 3.1 and [21], Lemma 3.9. For (3.14), we have from Lemma 3.1,

$$
|X u|^{r} \Delta_{X} u \leq \frac{m(k-1)}{m(k-1)-r(m-k)} \Delta_{p} u
$$

and since

$$
\int_{\Omega} \eta \Delta_{p} u \leq \int_{\Omega}|X \eta||X u|^{p-1}
$$

for any $\eta \geq 0, \in C_{0}^{1}(\Omega)$, we infer (3.14) from (3.13). 
By using the subelliptic Sobolev inequality [2], [11], we obtain corresponding $L^{p}$ estimates, namely

$$
\|u\|_{L^{p}\left(\Omega^{\prime}\right)} \leq C\|u\|_{L^{1}(\Omega)}
$$

where

$$
1 \leq p<\frac{Q k(m-1)}{(Q-1) m-(Q+m-2) k}
$$

for $(Q-1) m \geq(Q+m-2) k$ and $p=\infty$ if $(Q-1) m<(Q+m-2) k$. For this last case we have a Hölder estimate [12], [21],

$$
\sup _{\Omega^{\prime}} \frac{|u(x)-u(y)|}{|d(x, y)|^{\alpha}} \leq C\|u\|_{L^{1}(\Omega)}
$$

where

$$
\alpha=\frac{k(Q+m-2)-m(Q-1)}{k(m-1)},
$$

if $k<m(\alpha<1$ if $k=m)$.

\section{WeAk Continuity}

In this section, we complete the proof of Theorem 1.1. First we prove a local bound for $\mathscr{F}_{2}$. For convenience we use the notation

$$
\mathscr{E}_{2}[u]=\sum_{i<j}\left(\left[X_{i}, X_{j}\right] u\right)^{2},
$$

so that

$$
\mathscr{F}_{2}[u]=F_{2}[u]+\frac{3}{4} \mathscr{E}_{2}[u]
$$

Lemma 4.1. Let $u \in C^{2}(\Omega)$ be 2-convex in $\Omega$ with respect to $X_{1}, \ldots X_{m}$, satisfying hypotheses (i) to (iii). Then, for any subdomain $\Omega^{\prime} \subset \subset \Omega$, we have

$$
\int_{\Omega^{\prime}} \mathscr{F}_{2}[u] \leq C\left(\int_{\Omega}|u|\right)^{2},
$$

where $C$ depends on $\operatorname{dist}\left(\Omega^{\prime}, \partial \Omega\right), X_{1}, \ldots X_{m}$.

In particular, Lemma 4.1 provides a local $L^{2}$ estimate for the commutators $\left[X_{i}, X_{j}\right] u$. 
Proof. Letting $\eta>0 \in C_{0}^{1}(\Omega)$ be a cut-off function, we have

$$
\begin{aligned}
\int_{\Omega} \eta^{2} \mathscr{F}_{2}[u] & =\frac{1}{2} \int_{\Omega} \eta^{2} \mathscr{F}_{2}^{i j} X_{i} X_{j} u \\
& =-\frac{1}{2} \int_{\Omega} \mathscr{F}_{2}^{i j} X_{i} \eta^{2} X_{j} u \\
& =-\frac{1}{2} \int_{\Omega}\left\{F_{2}^{i j} X_{i} \eta^{2} X_{j} u+\frac{3}{2}\left[X_{i}, X_{j}\right] u X_{i} \eta^{2} X_{j} u\right\} \\
& \leq C \int_{\Omega}\left\{\left|X \eta^{2}\right||X u| \Delta_{X} u+\eta \mathscr{E}_{2}^{\frac{1}{2}}[u]|X \eta||X u|\right\} .
\end{aligned}
$$

Consequently,

$$
\int_{\Omega} \eta^{2} \mathscr{F}_{2}[u] \leq C \int_{\Omega}\left\{\left|X \eta^{2}\right||X u| \Delta_{X} u+|X \eta|^{2}|X u|^{2}\right\}
$$

and (4.3) follows from Theorem 3.1.

Proof of Theorem 1.1. Letting $u$ and $v$ be 2-convex in $\Omega, u_{t}=t u+(1-t) v, 0 \leq$ $t \leq 1$ and $\eta \geq 0, \in C_{0}^{2}(\Omega)$, we now have

$$
\begin{aligned}
& \int_{\Omega} \eta\left(\mathscr{F}_{2}[u]-\mathscr{F}_{2}[v]\right) \\
= & \int_{0}^{1} d t \int_{\Omega} \eta \mathscr{F}_{2}^{i j}\left[u_{t}\right] X_{i} X_{j}(u-v) \\
= & \frac{1}{2} \int_{\Omega} \eta \mathscr{F}_{2}^{i j}[u+v] X_{i} X_{j}(u-v) \\
= & -\frac{1}{2} \int_{\Omega} \mathscr{F}_{2}^{i j} X_{i} \eta X_{j}(u-v) \\
= & -\frac{1}{2} \int_{\Omega}\left\{\mathscr{F}_{2}^{j i}+3\left[X_{i}, X_{j}\right](u+v)\right\} X_{i} \eta X_{j}(u-v) \\
= & \frac{1}{2} \int_{\Omega}\left\{\mathscr{F}_{2}^{i j} X_{i} X_{j} \eta(u-v)-3\left[X_{i}, X_{j}\right](u+v) X_{i} \eta X_{j}(u-v)\right\} \\
= & \frac{1}{2} \int_{\Omega} F_{2}^{i j}[u+v]\left(X_{i} X_{j} \eta\right)(u-v) \\
& +\frac{3}{4} \int_{\Omega}\left[X_{i}, X_{j}\right](u+v)\left\{\left(X_{i} X_{j} \eta\right)(u-v)-2 X_{i} \eta X_{j}(u-v)\right\} \\
:= & \frac{1}{2} I_{1}+\frac{3}{4} I_{2}
\end{aligned}
$$


The estimation of the integral $I_{1}$ is similar to the corresponding term in the commuting case [19]. Namely

$$
\begin{aligned}
\left|I_{1}\right| & =\left|\int_{\Omega} F_{2}^{i j}[u+v]\left(X_{i} X_{j} \eta\right)(u-v)\right| \\
& \leq \int_{\Omega} \Delta_{X}(u+v)\left|X^{2} \eta\right||u-v| .
\end{aligned}
$$

Denoting

$$
\delta=\int_{\Omega}|u-v|, \quad K=\int_{\Omega}|u+v|,
$$

we have, for any $\epsilon>0$,

$$
|u-v|<\epsilon
$$

except on a set $A_{\epsilon}$ of measure $\left|A_{\epsilon}\right| \leq \delta / \epsilon$. We then estimate for a further cut-off function $\tilde{\eta} \in C_{0}^{1}(\Omega), 0 \leq \tilde{\eta} \leq 1$,

$$
\begin{aligned}
& \int_{\Omega} \tilde{\eta} \Delta_{X}(u+v)(u-v)^{+} \\
\leq & \epsilon \int_{\Omega} \tilde{\eta} \Delta_{X}(u+v)+\int_{\Omega} \tilde{\eta}(u-v-\epsilon)^{+} \Delta_{X}(u+v) \\
= & \epsilon \int_{\Omega} \tilde{\eta} \Delta_{X}(u+v)-\int_{A_{\epsilon}} X_{i}(u+v) X_{i}\left\{\tilde{\eta}(u-v-\epsilon)^{+}\right\} \\
\leq & C\left(\epsilon+\left|A_{\epsilon}\right|^{1-\frac{2}{q}}\right) \\
\leq & C\left\{\epsilon+\left(\frac{\delta}{\epsilon}\right)^{1-\frac{2}{q}}\right\}
\end{aligned}
$$

by Theorem 3.1, where $q$ is chosen so that

$$
2<q<\frac{2 Q(m-1)}{(Q-1)(m-2)}
$$

and $C$ depends on $\tilde{\eta}, K, \delta, X_{1}, \ldots X_{m}$. It then follows that

$$
\left|I_{1}\right| \leq C\left\{\epsilon+\left(\frac{\delta}{\epsilon}\right)^{1-\frac{2}{q}}\right\}
$$

where $C$ depends on $\eta, K, \delta, X_{1}, \ldots X_{m}$. To estimate $I_{2}$, we first use the bound for $\mathscr{E}_{2}$ in Lemma 4.1, to obtain

$$
I_{2} \leq C\left\{\int_{\operatorname{supp} \eta}|X(u-v)|^{2}+|u-v|^{2}\right\}^{\frac{1}{2}},
$$


where $C$ depends on $\eta, K, \delta, X_{1}, \ldots X_{m}$. The first part of the above integral may be estimated similarly to $I_{1}$, since

$$
\begin{aligned}
\int_{\Omega} \tilde{\eta}|X(u-v)|^{2} & =-\int_{\Omega} X_{i}\left(\tilde{\eta} X_{i}(u-v)\right)(u-v) \\
& \leq \int_{\Omega}\left\{|X \tilde{\eta}||X(u-v)|+\tilde{\eta} \Delta_{X}(u-v)\right\}|u-v| \\
& \leq \int_{\Omega} \tilde{\eta} \Delta_{X}(u-v)|u-v|+C\left\{\int_{\operatorname{supp} \tilde{\eta}}|u-v|^{2}\right\}^{\frac{1}{2}}
\end{aligned}
$$

while the second parts of (4.11) and (4.12) are handled readily by the estimate (3.17). It follows that

$$
I_{2} \leq C\left\{\epsilon+\left(\frac{\delta}{\epsilon}\right)^{1-\frac{2}{q}}\right\}^{\frac{1}{2}}
$$

With appropriate choice of $\delta$, we then conclude Theorem 1.1 from the estimates (4.10) and (4.13).

Remark. By inspection of the above proofs, we see that condition (iii) may be weakened to only requiring that the vector fields

$$
Y_{j}:=\sum_{i=1}^{m}\left[X_{i},\left[X_{i}, X_{j}\right]\right], \quad j=1 \ldots m,
$$

lie in the span of $X_{i},\left[X_{i}, X_{j}\right], i, j=1 \ldots m$. Without condition (iii) additional terms

$$
-\frac{1}{2} \int_{\Omega} \eta^{2} X u \cdot Y u
$$

and

$$
-\frac{1}{2} \int_{\Omega} \eta X(u-v) \cdot Y(u+v)+\frac{1}{2} \int_{\Omega}(u-v) X \eta \cdot Y(u+v)
$$

will arise in the right hand sides of (4.4) and (4.6) respectively and these are then automatically controlled. More general hypotheses are clearly possible. Note also that if $Y$ commutes with $X$, then the quantity

$$
\mathscr{F}_{2}^{*}[u]:=\mathscr{F}_{2}[u]+\frac{1}{2} X u . Y u
$$


also satisfies the monotonicity property (2.9). For the Engel group, this was first observed in [7]. More generally if we integrate by parts in (4.15), we find

$$
\begin{aligned}
& \int_{\Omega} \eta X(u-v) \cdot Y(u+v) \\
= & -\int_{\Omega} \eta(u+v) Y \cdot X(u-v)+(u+v) Y \eta \cdot X(u-v) \\
= & \int_{\Omega} \eta\{X(u+v) \cdot Y(u-v)+(u+v) Z(u-v)\} \\
& -\int_{\Omega}(u+v)\{Y \eta \cdot X(u-v)-X \eta \cdot Y(u-v)\}
\end{aligned}
$$

where $Z$ is the vector field given by

$$
Z=X . Y-Y . X=0 .
$$

Inserting (4.17) into (4.6) and (4.15) we may infer weak continuity results, in the absence of condition (iii), with respect to stronger topologies. For example $F_{2}$ will be weakly continuous with respect to $L_{l o c}^{1}(\Omega)$ on 2-convex functions, with uniformly bounded $X$ - and $Y$-gradients in $L_{l o c}^{\infty}(\Omega)$ and $L_{l o c}^{1}(\Omega)$ respectively.

Finally without hypothesis (iii) in Lemma 2.1, we obtain, from the proof,

$$
\begin{aligned}
\int_{\Omega}\left(\mathscr{F}_{2}[u]-\mathscr{F}_{2}[v]\right) & \geq-\frac{1}{2} \int_{\Omega} Y(u+v) \cdot X(u-v) \\
& =-\frac{1}{4} \int_{\Omega}\{Y(u+v) \cdot X(u-v)+Y(u-v) \cdot X(u+v)\}
\end{aligned}
$$

so that in general

$$
\int_{\Omega}\left(\mathscr{F}_{2}^{*}[u]-\mathscr{F}_{2}^{*}[v]\right) \geq 0 .
$$

See [5] for a more thorough analysis of monotonicity. In a similar fashion, we may combine (4.15) and (4.17) in (4.6) to conclude,

$$
\begin{aligned}
\int_{\Omega} \eta\left(\mathscr{F}_{2}^{*}[u]-\mathscr{F}_{2}^{*}[v]\right)= & \frac{1}{2} I_{1}+\frac{3}{4} I_{2}+\frac{3}{4} \int_{\Omega}(u-v) X \eta \cdot Y(u+v) \\
& -\frac{1}{4} \int_{\Omega}(u-v) X(u+v) . Y \eta
\end{aligned}
$$

\section{General $k$-COnvex functions}

For our purposes here, we define a function $u \in L_{l o c}^{1}(\Omega)$ to be $k$-convex in $\Omega$, with respect to the system of vector fields $X=\left(X_{1} \ldots X_{m}\right)$ if, for any $\Omega^{\prime} \subset \subset$ $\Omega$, there exists a sequence of $k$-convex functions in $C^{2}\left(\Omega^{\prime}\right)$ converging to $u$ in $L_{l o c}^{1}\left(\Omega^{\prime}\right)$. We designate the general class of $k$-convex functions in $\Omega$ by $\phi^{k}(\Omega)$. The estimates of the preceeding sections then extend as regularity properties and 
imbeddings of $\phi^{k}(\Omega)$. In particular if the system $X$ satisfies conditions (i) and (ii), we have from Theorem 3.1 that the distributional derivatives $X u \in L_{l o c}^{q}(\Omega)$ for

$$
1 \leq q<\frac{Q k(m-1)}{(Q-1)(m-k)}
$$

and that $\phi^{k}(\Omega)$ imbeds continuously in the Sobolev space,

$$
S_{l o c}^{1, q}(\Omega)=\left\{u \in L_{l o c}^{1}(\Omega) \mid X u \in L_{l o c}^{q}(\Omega)\right\},
$$

as defined, for example, in [2], [21]. Moreover for

$$
k>\frac{(Q-1) m}{(Q+m-2)}
$$

we see from (3.18) that $\phi^{k}(\Omega)$ imbeds continuously in the Hölder space $C^{0, \alpha}(\Omega)$ where

$$
\alpha=\frac{k(Q+m-2)-m(Q-1)}{k(m-1)},
$$

if $k<m(\alpha<1$ if $k=m)$. It follows from [14] that we can also take $\alpha=1$ when $k=m$ and $X$ generates the Lie algebra of a Carnot group.

For $k \geq 2$, the symmetric Hessian $X_{s}^{2} u$ consists of signed Radon measures. This follows exactly as in the Euclidean case [3], [19] but it may also be observed directly from the degenerate ellipticity of $F_{2}$,

$$
\Delta_{X} u I-X_{s}^{2} u \geq 0 \text {. }
$$

By taking limits, we see that the above quantities are measures if $u \in \phi^{k}(\Omega)$. If we also assume $X$ satisfies (iii), we obtain from Lemma 4.1, that the commutators $\left[X_{i}, X_{j}\right] u \in L_{l o c}^{2}(\Omega)$ for $u \in \phi^{k}(\Omega), k \geq 2$. Hence the full Hessian $X^{2} u$ comprises Radon measures. From Theorem 3.1, we also infer that $\phi^{k}(\Omega), k \geq 2$, imbeds continuously into the Euclidean Sobolev space $W_{l o c}^{1,2}(\Omega)$ if condition (iii) is strengthened to all second order commutators vanishing. Also if $X$ generates the Lie algebra of a Carnot group as in [1], then from the Hölder estimate (3.18) and the weak differentiability result in [1] we conclude that functions in $\phi^{k}(\Omega)$ will be twice differentiable almost everywhere in $\Omega$, with respect to the system $X$, if $k$ satisfies (5.3). This extends the corresponding result for the convex case $k=m$ in [5], [9], [14] and the Euclidean case $k>n / 2$ in [3].

Finally we may define the Hessian measure $\mu_{2}[u]$, with respect to $X$, for any function $u \in \phi^{2}(\Omega)$ by

$$
\int_{\Omega} \eta d \mu_{2}[u]=\lim _{m \rightarrow \infty} \int_{\Omega} \eta d \mu_{2}\left[u_{m}\right]
$$

where $\eta \in C_{0}^{0}(\Omega)$, supp $\eta \subset \Omega^{\prime} \subset \subset \Omega$ and $\left\{u_{m}\right\} \subset C^{2}\left(\Omega^{\prime}\right)$ is a sequence of $k$ convex functions converging to $u$ in $L_{l o c}^{1}\left(\Omega^{\prime}\right)$. By Theorem $1.1 \mu_{2}$ is well defined 
and weakly continuous with respect to convergence in $L_{l o c}^{1}(\Omega)$, that is if $\left\{u_{m}\right\} \subset$ $\phi^{2}(\Omega)$, converges to $u \in \phi^{2}(\Omega)$ in $L_{l o c}^{1}(\Omega)$, the corresponding sequence of measures $\left\{\mu_{2}\left[u_{m}\right]\right\}$ converges weakly to $\mu_{2}[u]$. Note that the case $\alpha=\frac{1}{2}$ in Theorem 1.1 also shows that the sum of the principal $2 \times 2$ minors of the full Hessian $X^{2} u$ also extends as a weakly continuous measure on $\phi^{2}(\Omega)$. From Lemma 2.1, we also conclude a more general monotonicity property, namely that if $u, v \in \phi^{2}(\Omega)$ satisfy $u \leq v$ in $\Omega, u=v$ continuously on $\partial \Omega$, then

$$
\mu_{2}[v](\Omega)+\frac{3}{4} \mathscr{E} 2[v] \leq \mu_{2}[u](\Omega)+\frac{3}{4} \mathscr{E}_{2}[u]
$$

General subharmonic functions. More generally we may define subharmonic functions along the lines of [19], [20], [21]. In particular we define an uppersemicontinuous function $u: \Omega \mapsto[-\infty, \infty)$ to be subharmonic with respect to the operator $F_{k}$ if $u$ satisfies $F_{k}[u] \geq 0$ in the viscosity sense, that is for any quadratic polynomial $q$ for which the difference $u-q$ has a finite local maximum at a point $y \in \Omega$, we have $F_{k}[q](y) \geq 0$. For smooth vector fields and $k=1$, this is also equivalent to our definition in [21], which corresponds to the traditional definition of subharmonicity. A $k$-convex function, as defined above by approximation, will be equivalent to a subharmonic function and moreover the estimates of Section 3 extend to the class of proper subharmonic functions. For Carnot groups of step 2, it follows from [14] that proper subharmonic functions will also be $k$-convex but we would expect this characterization to hold more generally. The equivalence of various definitions in the convex case, $k=m$, for Carnot groups is treated in the papers [4], [10], [13], [14].

\section{REFERENCES}

[1] L. Ambrosio and V. Magnani. Weak differentiability of BV functions on stratified groups. Math. Zeit., (245):123 - 153, 2003.

[2] L. Capogna, D. Danielli, and N. Garofalo. An embedding theorem and the Harnack inequality for nonlinear subelliptic equations. Comm. Part. Diff. Eqns, (18):1765 - 1794, 1993.

[3] N. Chaudhuri and N.S. Trudinger. An Alexsandrov type theorem for $k$-convex functions. Bull. Aust. Math. Soc., 71:305-314, 2005.

[4] D. Danielli, N. Garofalo, and D.M. Nhieu. Notions of convexity in Carnot groups. Comm. Anal. Geom., 11(2):263-341, 2003.

[5] D. Danielli, N. Garofalo, D.M. Nhieu, and F. Tournier. The theorem of Busemann-FellerAlexandrov in Carnot groups. Comm. Anal. Geom, 12(4):853-886, 2004.

[6] G.B. Folland and E.M. Stein. Hardy spaces on homogeneous groups. Princeton Univ. Press, 1982.

[7] N. Garofalo and F. Tournier. New properties of convex functions in the Heisenberg group. Trans. Amer. Math. Soc., to appear.

[8] C.E. Gutiérrez and A. Montanari. Maximum and comparison principles for convex functions on the Heisenberg group. Comm. Part. Diff. Eqns., 29:1305-1334, 2004.

[9] C.E. Gutiérrez and A. Montanari. On the second order derivatives of convex functions on the Heisenberg group. Ann. Scuola Norm. Sup. Pisa Cl. Sci (5), 3(2):349-366, 2004. 
[10] P. Juutinen, G. Lu, J.J. Manfredi, and B. Stroffolini. Convex functions on Carnot groups, (preprint)

[11] G. Lu. Weighted Poncaré and Sobolev inequalities for vector fields statisfying Hormander's condition and applications. Rev. Mat. Iberoamericana 8:367-439, 1992.

[12] G. Lu. Embedding theorems into Lipschitz and BMO spaces and applications to quasilinear subelliptic differential equations. Publ. Mat. 40:301-329, 1996.

[13] G. Lu, J.J. Manfredi and B. Stroffolini. Convex functions on the Heisenberg Group. Calc. Var. Partial Differential Equations 19:1-22, 2004.

[14] V. Magnani. Lipschitz continuity, Aleksandrov theorem and characterizations for H-convex functions. Math. Annaler(to appear).

[15] A. Nagel, E.M. Stein, and S. Wainger. Balls and metrics defined by vector fields. Acta. Math., 155:103-147, 1985.

[16] A.V. Pogorelov. Monge-Ampére equations of elliptic type. Noordhoff, Groningen, 1964.

[17] E.M. Stein. Harmonic Analysis. Princeton Univ Press, 1993.

[18] N.S. Trudinger and X.J. Wang. Hessian Measures I. Topol. Methods Nonlinear Anal., 10:225-239, 1997.

[19] N.S. Trudinger and X.J. Wang. Hessian Measures II. Ann. Math, 150:579-604, 1999.

[20] N.S. Trudinger and X.J. Wang. Hessian Measures III. J. Funct. Anal., 193:1-23, 2002.

[21] N.S. Trudinger and X.J. Wang. On the weak continuity of elliptic operators and applications to potential theory. Amer. J. Math, 124:369-410, 2002.

Neil S Trudinger

Centre for Mathematics and its Applications

Australian National University

Canberra, ACT 0200, Australia.

Email: neil.trudinger@anu.edu.au 